\title{
An Efficient Optimization Method for Solving Unsupervised Data Classification Problems
}

\author{
Parvaneh Shabanzadeh ${ }^{1,2}$ and Rubiyah Yusof ${ }^{1,2}$ \\ ${ }^{1}$ Centre for Artificial Intelligence and Robotics, Universiti Teknologi Malaysia, 54100 Kuala Lumpur, Malaysia \\ ${ }^{2}$ Malaysia-Japan International Institute of Technology (MJIIT), Universiti Teknologi Malaysia, 54100 Kuala Lumpur, Malaysia
}

Correspondence should be addressed to Rubiyah Yusof; rubiyah.kl@utm.my

Received 13 March 2015; Revised 11 June 2015; Accepted 29 June 2015

Academic Editor: Andrzej Kloczkowski

Copyright (C) 2015 P. Shabanzadeh and R. Yusof. This is an open access article distributed under the Creative Commons Attribution License, which permits unrestricted use, distribution, and reproduction in any medium, provided the original work is properly cited.

Unsupervised data classification (or clustering) analysis is one of the most useful tools and a descriptive task in data mining that seeks to classify homogeneous groups of objects based on similarity and is used in many medical disciplines and various applications. In general, there is no single algorithm that is suitable for all types of data, conditions, and applications. Each algorithm has its own advantages, limitations, and deficiencies. Hence, research for novel and effective approaches for unsupervised data classification is still active. In this paper a heuristic algorithm, Biogeography-Based Optimization (BBO) algorithm, was adapted for data clustering problems by modifying the main operators of $\mathrm{BBO}$ algorithm, which is inspired from the natural biogeography distribution of different species. Similar to other population-based algorithms, BBO algorithm starts with an initial population of candidate solutions to an optimization problem and an objective function that is calculated for them. To evaluate the performance of the proposed algorithm assessment was carried on six medical and real life datasets and was compared with eight well known and recent unsupervised data classification algorithms. Numerical results demonstrate that the proposed evolutionary optimization algorithm is efficient for unsupervised data classification.

\section{Introduction}

Unsupervised data classification (or data clustering) is one of the most important and popular data analysis techniques and refers to the process of grouping a set of data objects into clusters, in which the data of a cluster must have high degree of similarity and the data of different clusters must have high degree of dissimilarity [1]. The aim is to minimize the intercluster distance and maximize the intracluster distance. Clustering techniques have been applied in many areas such as document clustering $[2,3]$, medicine $[4,5]$, biology [6], agriculture [7], marketing and consumer analysis [8, 9], geophysics [10], prediction [11], image processing [12-14], security and crime detection [15], and anomaly detection [16].

In clustering problem, a dataset is divided into $k$ number of subgroups such that elements in one group are more similar to one another than elements of another group [17]. It can be defined to find out unknown patterns, knowledge, and information from a given dataset $A$ which was previously undiscovered using some criterion function [18]. It is NP complete problem when the number of cluster is greater than three [17]. Over the last two decades, many heuristic algorithms have been suggested and it is demonstrated that such algorithms are suitable for solving clustering problems in large datasets. For instance, the Tabu Search Algorithm for the clustering is presented in [19], the Simulated Annealing Algorithm in [20], the Genetic Algorithm in [21], and the particle swarm optimization algorithm in [22], which is one of powerful optimization methods. Fernández Martínez and Garcia-Gonzalo [23-26] clearly explained how PSO family parameters should be chosen close to the second order stability region. Hatamlou et al. in [27] introduced the Big Bang Big Crunch algorithm for the clustering problem. This algorithm has its origin from one of the theories of the evolution of the universe, namely, the Big Bang and Big Crunch theory. An Ant Colony Optimization was developed to solve the clustering problem in [28]. Such algorithms are 
able to find the global solution to the clustering. Application of the Gravitational Search Algorithm (GSA) [29] for clustering problem has been introduced in [30]. A comprehensive review on clustering algorithms can be found in [3133].

In this paper, a new heuristic clustering algorithm is developed. It is based on the evolutionary method called the Biogeography-Based Optimization (BBO) method proposed in [34]. The BBO method is inspired from the science of biogeography; it is a population-based evolutionary algorithm. Convergence results for this method and its practical applications can be found in [35]. The algorithm has demonstrated good performance on various optimization benchmark problems [36]. The proposed clustering algorithm is tested on six datasets from UCI Machine Learning Repository [37] and the obtained results are compared with those obtained using other similar algorithms.

The rest of this paper is organized as follows. Section 2 describes clustering problem. A brief overview of the $\mathrm{BBO}$ algorithm is given in Section 3. Section 4 presents the clustering algorithm. Experimental results are reported in Section 5. Finally, Section 6 presents conclusions with future research direction.

\section{Cluster Analysis}

In cluster analysis we suppose that we have been given a set $A$ of a finite number of points of $d$-dimensional space $R^{d}$, that is $\left\{a^{1}, a^{2}, \ldots, a^{n}\right\}$, where $a^{i} \in R^{d}, i=1,2, \ldots, n$.

In all, clustering algorithms can be classified into two categories, namely, hierarchical clustering and partitional clustering. Partitional clustering methods are the most popular class of center based clustering methods. It has been seen that partitional algorithm is more commendable rather than hierarchical clustering. The advantage of partitional algorithm is its visibility in circumstances where application involving large dataset is used where construction of nested grouping of patterns is computationally prohibited [38, 39]. The clustering problem is said to be hard clustering if every data point belongs to only one cluster. Unlike hard clustering, in the fuzzy clustering problem the clusters are allowed to overlap and instances have degrees of appearance in each cluster [40]. In this paper we will exclusively consider the hard unconstrained clustering problem. Therefore, the subject of cluster analysis is the partition of the set $A$ into a given number $q$ or disjoint subsets $B_{i}, i=1,2, \ldots, q$, with respect to predefined criteria such that

$$
\begin{aligned}
B_{i} & \neq \emptyset, \quad i=1,2, \ldots, q, \\
B_{i} \cap B_{j} & =\emptyset, \quad \forall i \neq j, i, j=1,2, \ldots, q, \\
\bigcup_{i=1}^{q} B_{i} & =A .
\end{aligned}
$$

Each cluster $B_{i}$ can be identified by its center (or centroid). To determine the dissimilarity between objects, many distance metrics have been defined. The most popular distance metric is the Euclidean distance. In this research we will also use Euclidean metric as a distance metric to measure the dissimilarity between data objects. So, for given two objects $a^{i}$ and $a^{j}$ with $d$-dimensions, the distance is defined by [38] as

$$
d\left(a^{i}, a^{j}\right)=\sqrt{\sum_{r=1}^{d}\left(a_{i}^{r}-a_{j}^{r}\right)^{2}}
$$

Since there are different ways to cluster a given set of objects, a fitness function (cost function) for measuring the goodness of clustering should be defined. A famous and widely used function for this purpose is the total mean-square quantization error (MSE) [41], which is defined as follows:

$$
\operatorname{MSE}=\sum_{j=1}^{q} \sum_{a^{i} \in B_{j}} d\left(a^{i}, B_{j}\right)^{2},
$$

where $d\left(a^{i}, B_{j}\right)^{2}$ is the distance between object $a^{i}$ and the center of cluster $C_{j}\left(B_{j}\right)$ to be found by calculating the mean value of objects within the respective cluster.

\section{Biogeography-Based Optimization Algorithm}

In this section, we give a brief description of the Biogeography-Based Optimization (BBO) algorithm. BBO is a new evolutionary optimization method based on the study of geographic distribution of biological organisms (biogeography) [34]. Organisms in BBO are called species, and their distribution is considered over time and space. Species can migrate between islands which are called habitat. Habitat is characterized by a Habitat Suitability Index (HSI). HSI in $\mathrm{BBO}$ is similar to the fitness in other population-based optimization algorithms and measures the solution goodness. HSI is related to many features of the habitat [34]. Considering a global optimization problem and a population of candidate solutions (individuals), each individual can be considered as a habitat and is characterized by its HSI. A habitat with high HSI is a good solution (maximization problem). Similar to other evolutionary algorithms, good solutions share their features with others to produce a better population in the next generations. Conversely, an individual with low fitness is unlikely to share features and likely accept features. Suitability index variable (SIV) implies the habitability of a habitat. As there are many factors in the real world which make a habitat more suitable to reside than others, there are several SIVs for a solution which affect its goodness. A SIV is a feature of the solution and can be imagined like a gene in GA. BBO consists of two main steps: migration and mutation. Migration is a probabilistic operator that is intended to improve a candidate solution $[42,43]$. In $\mathrm{BBO}$, the migration operator includes two different types: immigration and emigration, where for each solution in each 
generation, the rates of these types are adaptively determined based on the fitness of the solution. In BBO, each candidate solution $h_{i}$ has its own immigration rate $\lambda_{i}$ and emigration rate $\mu_{i}$ as follows:

$$
\begin{aligned}
& \lambda_{i}=I\left(1-\frac{k(i)}{n \mathrm{pop}}\right), \\
& \mu_{i}=E\left(\frac{k(i)}{n \mathrm{pop}}\right),
\end{aligned}
$$

where $n$ pop is the population size and $k(i)$ shows the rank of $i$ th individual in a ranked list which has been sorted based on the fitness of the population from the worst fitness to the best one ( 1 is worst and $n$ pop is best). Also $E$ and $I$ are the maximum possible emigration and immigration rates, which are typically set to one. A good candidate solution has latively high emigration rate and allows immigration rate, while the converse is true for a poor candidate solution. Therefore if a given solution $h_{i}$ is selected to be modified (in migration step), then its immigration rate $\lambda_{i}$ is applied to probabilistically modify each SIV in that solution. The emigrating candidate solution $h_{j}$ is probabilistically chosen based on $\mu_{j}$. Different methods have been suggested for sharing information between habitats (candidate solutions), in [44], where migration is defined by

$$
h_{i}(\mathrm{SIV})=\alpha * h_{i}(\mathrm{SIV})+(1-\alpha) * h_{j}(\mathrm{SIV}) \text {, }
$$

where $\alpha$ is a number between 0 and 1 . It could be random or deterministic or it could be proportional to the relative fitness of the solutions $h_{i}$ and $h_{j}$. Equation (5) means that (feature solution) SIV of $h_{i}$ comes from a combination of its own SIV and the emigrating solution's SIV. Mutation is a probabilistic operator that randomly modifies a decision variable of a candidate solution. The purpose of mutation is to increase diversity among the population. The mutation rate is calculated in [34]

$$
m_{i}=m_{\max }\left(\frac{1-P_{i}}{P_{\max }}\right),
$$

where $P_{i}$ is the solution probability and $P_{\max }=\max _{i} P_{i}, i=$ $1, \ldots, n$ pop, where $n$ pop is the population size and $m_{\max }$ is user-defined parameter.

If $h_{i}(\mathrm{SIV})$ is selected for mutation, then the candidate solution $h_{j}$ is probabilistically chosen based on $m_{i}$; thus replace $h_{i}(\mathrm{SIV})$ with a randomly generated SIV. Several options can be used for mutation but one option for implementing that can be defined as

$$
h_{i}(\mathrm{SIV})=h_{i}(\mathrm{SIV})+\rho,
$$

where

$$
\rho=\partial\left(\max \left(h_{i}(\mathrm{SIV})\right)-\min \left(h_{i}(\mathrm{SIV})\right)\right) \sigma .
$$

\begin{tabular}{|l|l|l|l|l|l|}
\hline$C_{1}^{1}$ & $C_{1}^{2}$ & $C_{2}^{1}$ & $C_{2}^{2}$ & $C_{3}^{1}$ & $C_{3}^{2}$ \\
\hline
\end{tabular}

Figure 1: The encoding of an example of candidate solution.

$\partial$ is user-defined parameter near 0 and also $\max \left(h_{i}(\mathrm{SIV})\right)$, $\min \left(h_{i}(\mathrm{SIV})\right)$ are the upper and lower bounds for each decision variable and $\sigma$ is random number, normally distributed in the range of $(0,1)$.

Based on the above description, the main steps of the BBO algorithm can be described as follows.

Step 1 (initialization). At first, introduce the initial parameters that include the number of generations, necessary for the termination criterion, population size, which indicates the number of habitats/islands/solutions, number of design variables, maximum immigration and emigration rates, and mutation coefficient and also create a random set of habitats (population).

Step 2 (evaluation). Compute corresponding HSI values and rank them on the basis of fitness.

Step 3 (update parameters). Update the immigration rate $\lambda_{i}$ and emigration rate $\mu_{i}$ for each island/solution. Bad solutions have low emigration rates and high immigration rates whereas good solutions have high emigration rates and low immigration rates.

Step 4 (select islands). Probabilistically select the immigration islands based on the immigration rates and select the emigrating islands based on the emigration rates via roulette wheel selection.

Step 5 (migration phase). Randomly change the selected features (SIVs), based on (4)-(5) and based on the selected islands in the previous step.

Step 6 (mutation phase). Probabilistically carry out mutation based on the mutation probability for each solution, that is, based on (6).

Step 7 (check the termination criteria). If the output of the termination criterion step is not met, go to Step 2; otherwise, terminate it.

\section{BBO Algorithm for Data Clustering}

In order to use $\mathrm{BBO}$ algorithm for data clustering, onedimensional arrays are used to encode the centres of the desired clusters to present candidate solutions in the proposed algorithm. The length of the arrays is equal to $q \times d$, where $q$ is the number of clusters and $d$ is the dimensionality of the considered datasets. Figure 1 presents an example of candidate solution for a problem with 3 centroids clusters and 2 attributes. 
Then assume $\mathrm{POP}_{i}=\left\{C_{1}, C_{2}, \ldots, C_{q}\right\}$ is the $i$ th candidate solution and $C_{j}=\left\{C_{j}^{1}, C_{j}^{2}, \ldots, C_{j}^{d}\right\}$ is the $j$ th cluster centre for the $i$ th candidate solution $(i=1,2, \ldots, n$ pop) and $(j=$ $1,2, \ldots, q)$, so that $n$ pop is the number of islands or candidate solutions in which its value in this work is set to 100 . Therefore each of these candidate solutions shows centers of all clusters.

A good initial population is important to the performance of $\mathrm{BBO}$ and most of the population-based methods are affected by the quality of the initial population. Then in the proposed algorithm, taking into considering the nature of the input datasets, a high-quality population is created based on special ways as mentioned in pseudocodes. One of the candidate solutions will be produced by dividing whole dataset to $q$ equal sets, and three of them will be produced based on minimum, maximum, and average values of data objects in each dataset and other solutions will be created randomly. This procedure creates a high-quality initial population and consequently this procedure ensures that the candidate solutions are spread in the wide area of the search space, which as a result increases the chance of finding (near) global optima.

To ensure that the best habitats/solutions are preserved, elitist method is used to save the best individual found so far into the new population. So elitism strategy is proposed in order to retain the best solutions in the population from one generation to the next. Therefore in the proposed algorithm, new population is created based on merging initial population (old population) and the population due to migration and mutation process (new population). Then suppose POP is the entire initial population of candidate solutions and New POP is the initial population, changed by iteration of $\mathrm{BBO}$, and $\gamma$ is percentage of initial population that is chosen in next iteration (whose value in this work is 30\%). So the number of kept habitats of old population (KHOP) is as follows:

$$
\mathrm{KHOP}=\operatorname{round}(\gamma \times n \mathrm{pop}) .
$$

And the number of kept habitats of new population (KHCP) is as follows:

$$
\mathrm{KHCP}=n \mathrm{pop}-\mathrm{KHOP}
$$

Hence the population of next iteration can be as follows:

$$
\mathrm{POP} \longleftarrow\left[\begin{array}{c}
\operatorname{POP}(1: \mathrm{KHOP}) \\
\operatorname{NewPOP}(1: \mathrm{KHCP})
\end{array}\right]
$$

Suppose $\mathrm{POP}_{i}$ is the $i$ th candidate solution and $\operatorname{POP}_{i}(s)$ is the $s$ th decision variable of $\mathrm{POP}_{i}$ (i.e. $C_{r}^{t}, t=$ $1,2, \ldots, d$ and $r=1,2, \ldots, q)$. Based on the above description, the pseudocode of the proposed method is shown in Algorithm 1.
TABLE 1: Summarized characteristics of the test datasets.

\begin{tabular}{lccc}
\hline $\begin{array}{l}\text { Name of } \\
\text { dataset }\end{array}$ & $\begin{array}{c}\text { Number of data } \\
\text { objects }\end{array}$ & $\begin{array}{c}\text { Number of } \\
\text { features }\end{array}$ & $\begin{array}{c}\text { Number of } \\
\text { clusters }\end{array}$ \\
\hline Cancer & 683 & 9 & $2(444,239)$ \\
CMC & 1473 & 9 & $3(629,334,510)$ \\
Glass & 214 & 9 & $6(70,76,17,13,9$, \\
Iris & 150 & 4 & $3(50,50,50)$ \\
Vowel & 871 & 3 & $6(72,89,172,151$, \\
Wine & 178 & 13 & $207,180)$ \\
\hline
\end{tabular}

\section{Experimental Results}

The proposed method is implemented using MATLAB 7.6 on a T6400, $2 \mathrm{GHz}, 2 \mathrm{~GB}$ RAM computer. To evaluate the performance of the proposed algorithm, the results obtained have been compared with other algorithms by applying them on some well known datasets taken from Machine Learning Laboratory [37]. Six datasets are employed to validate the proposed method. These datasets named Cancer, CMC, Iris, Glass, Wine, and Vowel cover examples of data of low, medium, and high dimensions. The brief of the characteristics of these datasets is presented in Table 1. They have been applied by many authors to study and evaluate the performance of their algorithms, and they can be described as follows.

Wisconsin Breast Cancer Dataset $(n=683, d=9, k=$ 2 ). This dataset has 683 points with nine features such as cell size uniformity, clump thickness cell, bare nuclei, shape uniformity, marginal adhesion, single epithelial cell size, bland chromatin, normal nucleoli, and mitoses. There are two clusters in this dataset: malignant and benign.

Contraceptive Method Choice Dataset (denoted as CMC with $n=1473, d=10, k=3$ ). This dataset is a subset of the 1987 National Indonesia Contraceptive Prevalence Survey. The samples are married women who either were not pregnant or did not know if they were at the time of interview. The problem is to predict the choice of current contraceptive method (no use has 629 objects, long-term methods have 334 objects, and short-term methods have 510 objects) of a woman based on her demographic and socioeconomic characteristics.

Ripley's Glass Dataset ( $n=214, d=9, k=6)$. This dataset has 214 points with nine features. The dataset has six different clusters which are building windows float processed, building windows nonfloat processed, vehicle windows float processed, containers, tableware, and headlamps [41].

Iris Dataset ( $n=150, d=4, k=3$ ). This data consists of three different species of iris flower: Iris setosa, Iris virginica, and Iris versicolour. For each species, 50 samples with four 
Create an initial population POP, as follow:

(i) $\operatorname{POP}_{1}=\left\{C_{j} \mid C_{j}=\operatorname{Min}(\right.$ dataset $)-(j-1) *((\operatorname{Max}($ dataset $)-\operatorname{Min}($ dataset $\left.)) / q), j=1,2, \ldots, q\right\}$

Where Min(dataset) and $\operatorname{Max}$ (dataset) are correspond with data items that their features are the minimum and maximum values in whole of the dataset respectively.

(ii) $\mathrm{POP}_{2}=$ Create a candidate solution using the minimum of the dataset

(iii) $\mathrm{POP}_{3}=$ Create a candidate solution using the maximum of the dataset

(iv) $\mathrm{POP}_{4}=$ Create a candidate solution using the mean of the dataset

(v) $\mathrm{POP}_{5}, \ldots, \mathrm{POP}_{n \text { pop }}$ : Create all other candidate solutions randomly as follow:

for $i=5: n$ pop

for $j=1: d$

for $g=0: q-1$

$\operatorname{POP}_{i}(j+g * d)=$ random number in range of $(L C(j), U C(j))$

Where $L C(j)$ and $U C(j)$ are the lower and upper bounds for each decision

variable (i.e. $\left.L C(j)<C_{r}^{j}<U C(j), r=1, \ldots, q\right)$.

end for

end for

end for

Calculate fitness of POP (cost function) according to (5) and sort it from the Best

(minimum) fitness to the worst one (maximum).

for $i=1$ to $n$ pop, (for each solution $\mathrm{POP}_{i}$ )

Compute emigration rate $\mu_{i}$ proportional to fitness of $\mathrm{POP}_{i}$, where $\mu_{i} \in \operatorname{rand}[0,1]$, so that $\mu_{1}=1$,

$\mu_{\text {npop }}=0$ and immigration rate $\lambda_{i}=1-\mu_{\mathrm{i}}$, so that $\lambda_{1}=0, \lambda_{\text {npop }}=1$.

end for

Set $\partial, \gamma$, KHOP, KHCP based on (11)-(14)

While termination conditions are not met

for $i=1$ to $n$ pop (for each solution $\mathrm{NewPOP}_{i}$ )

for $s=1$ to $d$ (for each candidate solution decision variable index $s$ )

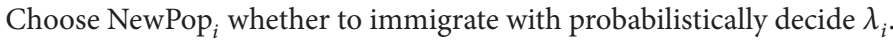

if $\mathrm{NewPOP}_{i}$ is selected for immigrating

Use values $\{\mu\}$ to probabilistically select the emigrating solution $\mathrm{POP}_{j}$.

if $\mathrm{POP}_{j}$ is selected for emigrating

$\operatorname{NewPOP}_{i}(s) \leftarrow \operatorname{POP}_{i}(s)+\alpha \times\left(\mathrm{POP}_{j}(s)-\mathrm{POP}_{i}(s)\right)$, based on (8) by setting

$\alpha=$ random number in range of $(0.9,1)$.

end if

end if

With probabilistically $m_{i}$ decide whether to mutate NewPOP ${ }_{i}$ based on (9).

if $\mathrm{NewPOP}_{i}$ is selected for mutation, thus based on (10), (11):

for $g=1: q$

if $(s-(g-1) * d)<=d$

$\operatorname{NewPOP}_{i}(s)=\operatorname{NewPOP}_{i}(s)+\sigma \times \operatorname{sigma}^{*}(s-(g-1))$

$\left\{{ }^{*} \operatorname{sigma}(j)=\partial \times(U C(j)-L C(j)), j=1, \ldots, d\right.$ where $\partial$ its value in this work Break

is 0.02 , also $U C(j)-L C(j)$ are the lower and upper bounds for each decision variable

end if

end for

end if

end for (repeat for next candidate solution decision variable index)

Recalculate fitness of NewPOP.

End for (repeat for next solution)

Sort population based on fitness function.

Make new population POP, based on combinatorial of old POP and New POP based on (10).

Sort new population based on fitness function.

Update and store, best solution ever found.

end while

Algorithm 1: Pseudocodes of proposed method. 
TABLE 2: Intracluster distances for real life datasets.

\begin{tabular}{|c|c|c|c|c|c|c|c|c|c|c|}
\hline Dataset & Criteria & $K$-means & TS & SA & PSO & BB-BC & GA & GSA & $\mathrm{ACO}$ & $\mathrm{BBO}$ \\
\hline \multirow{4}{*}{ Cancer } & Average & 3032.2478 & 3251.37 & 3239.17 & 2981.7865 & 2964.3880 & 3249.46 & 2972.6631 & $3,046.06$ & 2964.3879 \\
\hline & Best & 2986.9613 & 2982.84 & 2993.45 & 2974.4809 & 2964.3875 & 2999.32 & 2965.7639 & $2,970.49$ & 2964.3875 \\
\hline & Worst & 5216.0895 & 3434.16 & 3421.95 & 3053.4913 & 2964.3890 & 3427.43 & 2993.2446 & $3,242.01$ & 2964.3887 \\
\hline & Std. & 315.1456 & 232.217 & 230.192 & 10.43651 & 0.00048 & 229.734 & 8.91860 & 90.50028 & 0.00036 \\
\hline \multirow{4}{*}{$\mathrm{CMC}$} & Average & 5543.4234 & 5993.59 & 5893.48 & 5547.8932 & 5574.7517 & 5756.59 & 5581.9450 & $5,819.1347$ & 5532.2550 \\
\hline & Best & 5542.1821 & 5885.06 & 5849.03 & 5539.1745 & 5534.0948 & 5705.63 & 5542.2763 & $5,701.9230$ & 5532.2113 \\
\hline & Worst & 5545.3333 & 5999.80 & 5966.94 & 5561.6549 & 5644.7026 & 5812.64 & 5658.7629 & $5,912.4300$ & 5532.432 \\
\hline & Std. & 1.5238 & 40.845 & 50.867 & 7.35617 & 39.4349 & 50.369 & 41.13648 & 45.634700 & 0.06480 \\
\hline \multirow{4}{*}{ Glass } & Average & 227.9779 & 283.79 & 282.19 & 230.49328 & 231.2306 & 255.38 & 233.5433 & 273.46 & 215.2097 \\
\hline & Best & 215.6775 & 279.87 & 275.16 & 223.90546 & 223.8941 & 235.50 & 224.9841 & 269.72 & 210.6173 \\
\hline & Worst & 260.8385 & 286.47 & 287.18 & 246.08915 & 243.2088 & 278.37 & 248.3672 & 280.08 & 233.9314 \\
\hline & Std. & 14.1389 & 4.19 & 4.238 & 4.79320 & 4.6501 & 12.47 & 6.13946 & 3.5848 & 3.525 \\
\hline \multirow{4}{*}{ Iris } & Average & 105.7290 & 97.8680 & 99.95 & 98.1423 & 96.7654 & 125.1970 & 96.7311 & 97.1715 & 96.5653 \\
\hline & Best & 97.3259 & 97.3659 & 97.45 & 96.8793 & 96.6765 & 113.9865 & 96.6879 & 97.1007 & 96.5403 \\
\hline & Worst & 128.4042 & 98.56949 & 102.01 & 99.7695 & 97.4287 & 139.7782 & 96.8246 & 97.8084 & 96.6609 \\
\hline & Std. & 12.3876 & 72.86 & 2.018 & 0.84207 & 0.20456 & 14.563 & 0.02761 & 0.367 & 0.0394 \\
\hline \multirow{4}{*}{ Vowel } & Average & $153,660.8071$ & 162108.53 & 161566.28 & $153,218.23418$ & $151,010.0339$ & 159153.49 & $152,931.8104$ & $159,458.1438$ & 149072.9042 \\
\hline & Best & $149,394.8040$ & 149468.26 & 149370.47 & $152,461.56473$ & $149,038.5168$ & 149513.73 & $151,317.5639$ & $149,395.602$ & 148967.2544 \\
\hline & Worst & $168,474.2659$ & 165996.42 & 165986.42 & $158,987.08231$ & $153,090.4407$ & 165991.65 & $155,346.6952$ & $165,939.8260$ & 153051.96931 \\
\hline & Std. & 4123.04203 & 2846.235 & 0.645 & 2945.23167 & 1859.3235 & 3105.544 & 2486.70285 & $3,485.3816$ & 137.7311 \\
\hline \multirow{4}{*}{ Wine } & Average & $16,963.0441$ & 16785.46 & $17,521.09$ & $16,316.2745$ & $16,303.4121$ & $16,530.53$ & $16,374.3091$ & $16,530.53381$ & 16292.6782 \\
\hline & Best & $16,555.6794$ & 16666.22 & $16,473.48$ & $16,304.4858$ & $16,298.6736$ & $16,530.53$ & $16,313.8762$ & $16,530.53381$ & 16292.6782 \\
\hline & Worst & $23,755.0495$ & 16837.54 & $18,083.25$ & $16,342.7811$ & $16,310.1135$ & $16,530.53$ & $16,428.8649$ & $16,530.53381$ & 16292.6782 \\
\hline & Std. & 1180.6942 & 52.073 & 753.084 & 12.60275 & 2.6620 & 0 & 34.67122 & 0 & 0 \\
\hline
\end{tabular}

TABLE 3: The obtained best centroids coordinate for Cancer data.

\begin{tabular}{lcc}
\hline Cancer data & Cluster 1 & Cluster 2 \\
\hline Feature A & 7.1156 & 2.8896 \\
Feature B & 6.6398 & 1.1278 \\
Feature C & 6.6238 & 1.2018 \\
Feature D & 5.6135 & 1.1646 \\
Feature E & 5.2402 & 1.9943 \\
Feature F & 8.0995 & 1.1215 \\
Feature G & 6.0789 & 2.0059 \\
Feature H & 6.0198 & 1.1014 \\
Feature I & 2.3282 & 1.0320 \\
\hline
\end{tabular}

features each (sepal length, sepal width, petal length, and petal width) were collected [45].

Vowel Dataset $(n=871, d=3, k=6)$. It consists of 871 Indian Telugu vowel sounds. The dataset has three features corresponding to the first, second, and third vowel frequencies and six overlapping classes [45].

Wine Dataset $(n=178, d=13, k=3)$. This dataset describes the quality of wine from physicochemical properties
TABLE 4: The obtained best centroids coordinate for $C M C$ data.

\begin{tabular}{lccc}
\hline CMC data & Cluster 1 & Cluster 2 & Cluster 3 \\
\hline Feature A & 43.6354 & 33.4957 & 24.4102 \\
Feature B & 3.0140 & 3.1307 & 3.0417 \\
Feature C & 3.4513 & 3.5542 & 3.5181 \\
Feature D & 4.582 & 3.6511 & 1.7947 \\
Feature E & 0.7965 & 0.7928 & 0.9275 \\
Feature F & 0.7629 & 0.6918 & 0.7928 \\
Feature G & 1.8245 & 2.0903 & 2.2980 \\
Feature H & 3.4355 & 3.29183 & 2.9754 \\
Feature I & 0.094 & 0.0573 & 0.037 \\
\hline
\end{tabular}

in Italy. There are 178 instances with 13 continues attributes grouped into 3 classes. There is no missing value for attributes.

In this paper the performance of the proposed algorithm is compared with recent algorithms reported in the literature, including $K$-means [38], TS [19], SA [20], PSO [22, 39], BBBC [27], GA [21], GSA [30], and ACO [46].

In this paper two criteria are used to measure the quality of solutions found by clustering algorithms:

(i) Sum of intracluster distances: The distance between each data vector in a cluster and the centroid of that 
TABLE 5: The obtained best centroids coordinate for Glass data.

\begin{tabular}{|c|c|c|c|c|c|c|}
\hline Glass data & Cluster 1 & Cluster 2 & Cluster 3 & Cluster 4 & Cluster 5 & Cluster 6 \\
\hline Feature A & 1.5260 & 1.5156 & 1.5228 & 1.5266 & 1.5203 & 1.5243 \\
\hline Feature B & 11.9759 & 13.0863 & 14.6577 & 13.2229 & 13.7277 & 13.8085 \\
\hline Feature C & 0.006 & 3.5272 & 0.0061 & 0.4232 & 3.5127 & 2.3414 \\
\hline Feature D & 1.0514 & 1.3618 & 2.2170 & 1.5242 & 1.0249 & 2.5919 \\
\hline Feature E & 72.0540 & 72.8710 & 73.2504 & 73.0610 & 71.9072 & 71.1423 \\
\hline Feature F & 0.2552 & 0.5768 & 0.0299 & 0.3865 & 0.2067 & 2.5749 \\
\hline Feature G & 14.3566 & 8.3588 & 8.6714 & 11.1471 & 9.4166 & 5.9948 \\
\hline Feature $\mathrm{H}$ & 0.1808 & 0.0046 & 1.047 & 0.00979 & 0.0281 & 1.3373 \\
\hline Feature I & 0.1254 & 0.0568 & 0.0196 & 0.1544 & 0.0498 & 0.2846 \\
\hline
\end{tabular}

cluster is calculated and summed up, as defined in (3). It is also the evaluation fitness in this paper. Clearly, the smaller the value is, the higher the quality of the clustering is.

(ii) Error rate (ER): It is defined as the number of misplaced points over the total number of points in the dataset as

$$
\mathrm{ER}=\frac{\left(\sum_{i=1}^{n \text { pop }}\left(\text { if }\left(B_{i}=C_{i}\right) \text { then } 0 \text { else } 1\right)\right)}{n \text { pop }} * 100
$$

where $n$ pop is the total number of data points and $B_{i}$ and $C_{i}$ denote the datasets of which the $i$ th point is a member before and after clustering, respectively.

Since all the algorithms are stochastic algorithms, therefore for each experiment 10 independent runs are carried out to indicate the stability and robustness of the algorithms for against with the randomized nature of the algorithms. The average, best (minimum), and worst (maximum) solutions and standard deviation of solutions of 10 runs of each algorithm are obtained by using algorithms on the datasets, which have been applied for comparison. This process ensures that the candidate solutions are spread in the wide area of the search space and thus increases the chance of finding optima.

Table 2 presents the intracluster distances obtained from the eight clustering algorithms for the datasets above. For the cancer dataset, the average, best, and worst solutions of BBO algorithm are 2964.3879, 2964.3875, and 2964.3887, respectively, which are much better than those of other algorithms except BB-BC which is the same as it. This means that it provides the optimum value and small standard deviation, when compared to those obtained by the other methods. For the CMC dataset, the proposed method reaches an average of 5532.2550, while other algorithms were unable to reach this solution. Also, the results obtained on the glass dataset show that $\mathrm{BBO}$ method converges to the optimum of 215.2097 in all of runs while the average solutions of the $k$-means, TS, SA, GA, PSO, BB-BC, GSA, and ACO, are 227.9779, 283.79, 282.19, 230.49328, 231.2306, 255.38, 233.5433, and 273.46, respectively. For the iris dataset, the average of solutions found by BBO is 96.5653 , while this value
TABLE 6: The obtained best centroids coordinate for Iris data.

\begin{tabular}{lccc}
\hline Iris data & Cluster 1 & Cluster 2 & Cluster 3 \\
\hline Feature A & 5.0150 & 5.9338 & 6.7343 \\
Feature B & 3.4185 & 2.7974 & 3.0681 \\
Feature C & 1.4681 & 4.4173 & 5.6299 \\
Feature D & 0.2380 & 1.4165 & 2.1072 \\
\hline
\end{tabular}

for the $k$-means, TS, SA, GA, PSO, BB-BC, GSA, and ACO, is $105.7290,97.8680,99.95,98.1423,96.7654,125.1970,96.7311$, and 97.1715 , respectively. As seen from the results for the vowel dataset, the $\mathrm{BBO}$ algorithm outperformed the $K$-means, TS, SA, GA, PSO, BB-BC, GSA, and ACO algorithms, with the average solution 149072.9042. For the Wine dataset, the BBO algorithm achieved the optimum value of 16292.6782 , which is significantly better than the other tested algorithms.

From Table 2, we can see that the BBO algorithm has achieved the good performance in terms of the average, best, and worst intercluster distances on these six datasets. It means that $\mathrm{BBO}$ can find good quality solutions.

The best centroids coordinates obtained by the $\mathrm{BBO}$ algorithm on the test dataset are shown in Tables 3-8. Finally, Table 9 shows the error rate values obtained by algorithms for real datasets. As seen from the results in Table 9, the BBO algorithm presents a minimum average error rate in all the real datasets. However, the topography of the cost function of clustering (3) has a valley shape; therefore the found solutions by these methods were not global. Therefore the experimental results in the tables demonstrate that the proposed method is one of practicable and good techniques for data clustering.

\section{Conclusions}

In summary, this paper presents a new clustering algorithm based on the recently developed BBO heuristic algorithm that is inspired by mathematical models of science of biogeography (study of the distribution of animals and plants over time and space).

To evaluate the performance of the $\mathrm{BBO}$ algorithm, it was tested on six real life datasets and compared with other eight clustering algorithms. The experimental results indicate 
TABLE 7: The obtained best centroids coordinate for Vowel data.

\begin{tabular}{lcccccc}
\hline Vowel data & Cluster 1 & Cluster 2 & Cluster 3 & Cluster 4 & Cluster 5 & Cluster 6 \\
\hline Feature A & 357.8349 & 375.8459 & 508.1135 & 407.9219 & 623.6778 & 439.6126 \\
Feature B & $2,291.6435$ & $2,148.4110$ & $1,838.2133$ & $1,0182.0145$ & $1,309.8038$ & 987.4300 \\
Feature C & $2,978.2399$ & $2,678.8524$ & $2,555.9085$ & $2,317.2847$ & $2,332.7767$ & $2,665.4154$ \\
\hline
\end{tabular}

TABLE 8: The obtained best centroids coordinates for Wine data.

\begin{tabular}{lccc}
\hline Wine data & Cluster 1 & Cluster 2 & Cluster 3 \\
\hline Feature A & 13.3856 & 12.7859 & 12.7093 \\
Feature B & 1.9976 & 2.3535 & 2.3219 \\
Feature C & 2.3150 & 2.4954 & 2.4497 \\
Feature D & 16.9836 & 19.5480 & 21.1983 \\
Feature E & 105.2124 & 98.9327 & 92.6449 \\
Feature F & 3.0255 & 2.0964 & 2.1366 \\
Feature G & 3.1380 & 1.4428 & 1.9187 \\
Feature H & 0.51050 & 0.31322 & 0.3520 \\
Feature I & 2.3769 & 1.7629 & 1.4966 \\
Feature J & 5.7760 & 5.8415 & 4.3213 \\
Feature K & 0.8339 & 1.1220 & 1.2229 \\
Feature L & 3.0686 & 1.9611 & 2.5417 \\
Feature M & 1137.4923 & 687.3041 & 463.8856 \\
\hline
\end{tabular}

TABLE 9: Error rates for real life datasets.

\begin{tabular}{lcccc}
\hline Dataset & $K$-means & PSO & GSA & BBO \\
\hline Cancer & 4.08 & 5.11 & 3.74 & 3.7 \\
CMC & 54.49 & 54.41 & 55.67 & 54.22 \\
Glass & 37.71 & 45.59 & 41.39 & 36.47 \\
Iris & 17.80 & 12.53 & 10.04 & 10.03 \\
Vowel & 44.26 & 44.65 & 42.26 & 41.36 \\
Wine & 31.12 & 28.71 & 29.15 & 28.65 \\
\hline
\end{tabular}

that the $\mathrm{BBO}$ optimization algorithm is suitable and useful heuristic technique for data clustering. In order to improve the obtained results, as a future work, we plan to hybridize the proposed approach with other algorithms and we intend to apply this method with other data mining problems.

\section{Conflict of Interests}

The authors declare that there is no conflict of interests regarding the publication of this paper.

\section{Acknowledgments}

The authors would like to thank the Ministry of Education Malaysia for funding this research project through a Research University Grant of Universiti Teknologi Malaysia (UTM), project titled "Based Syariah Compliance Chicken Slaughtering Process for Online Monitoring and Enforcement" (01G55). Also, thanks are due to the Research Management Center (RMC) of UTM for providing an excellent research environment in which to complete this work.

\section{References}

[1] W. A. Barbakh, Y. Wu, and C. Fyfe, "Review of clustering algorithms," in Non-Standard Parameter Adaptation for Exploratory Data Analysis, vol. 249 of Studies in Computational Intelligence, pp. 7-28, Springer, Berlin, Germany, 2009.

[2] X. Cai and W. Li, "A spectral analysis approach to document summarization: clustering and ranking sentences simultaneously," Information Sciences, vol. 181, no. 18, pp. 3816-3827, 2011.

[3] M. Carullo, E. Binaghi, and I. Gallo, "An online document clustering technique for short web contents," Pattern Recognition Letters, vol. 30, no. 10, pp. 870-876, 2009.

[4] W. Halberstadt and T. S. Douglas, "Fuzzy clustering to detect tuberculous meningitis-associated hyperdensity in CT images," Computers in Biology and Medicine, vol. 38, no. 2, pp. 165-170, 2008.

[5] L. Liao, T. Lin, and B. Li, "MRI brain image segmentation and bias field correction based on fast spatially constrained kernel clustering approach," Pattern Recognition Letters, vol. 29, no. 10, pp. 1580-1588, 2008.

[6] E. R. Hruschka, R. J. G. B. Campello, and L. N. de Castro, "Evolving clusters in gene-expression data," Information Sciences, vol. 176, no. 13, pp. 1898-1927, 2006.

[7] P. Papajorgji, R. Chinchuluun, W. S. Lee, J. Bhorania, and P. M. Pardalos, "Clustering and classification algorithms in food and agricultural applications: a survey," in Advances in Modeling Agricultural Systems, pp. 433-454, Springer US, New York, NY, USA, 2009.

[8] Y.-J. Wang and H.-S. Lee, "A clustering method to identify representative financial ratios," Information Sciences, vol. 178, no. 4, pp. 1087-1097, 2008.

[9] J. Li, K. Wang, and L. Xu, "Chameleon based on clustering feature tree and its application in customer segmentation," Annals of Operations Research, vol. 168, no. 1, pp. 225-245, 2009.

[10] Y.-C. Song, H.-D. Meng, M. J. O'Grady, and G. M. P. O’Hare, "The application of cluster analysis in geophysical data interpretation," Computational Geosciences, vol. 14, no. 2, pp. 263-271, 2010.

[11] G. Cardoso and F. Gomide, "Newspaper demand prediction and replacement model based on fuzzy clustering and rules," Information Sciences, vol. 177, no. 21, pp. 4799-4809, 2007.

[12] S. Das and S. Sil, "Kernel-induced fuzzy clustering of image pixels with an improved differential evolution algorithm," Information Sciences, vol. 180, no. 8, pp. 1237-1256, 2010.

[13] R. I. John, P. R. Innocent, and M. R. Barnes, "Neuro-fuzzy clustering of radiographic tibia image data using type 2 fuzzy sets," Information Sciences, vol. 125, no. 1-4, pp. 65-82, 2000.

[14] S. Mitra and P. P. Kundu, "Satellite image segmentation with shadowed C-means," Information Sciences, vol. 181, no. 17, pp. 3601-3613, 2011.

[15] T. H. Grubesic, "On the application of fuzzy clustering for crime hot spot detection," Journal of Quantitative Criminology, vol. 22, no. 1, pp. 77-105, 2006. 
[16] N. H. Park, S. H. Oh, and W. S. Lee, "Anomaly intrusion detection by clustering transactional audit streams in a host computer," Information Sciences, vol. 180, no. 12, pp. 2375-2389, 2010.

[17] A. J. Sahoo and Y. Kumar, "Modified teacher learning based optimization method for data clustering," in Advances in Signal Processing and Intelligent Recognition Systems, vol. 264 of Advances in Intelligent Systems and Computing, pp. 429-437, Springer, 2014.

[18] M. R. Garey, D. S. Johnson, and H. S. Witsenhausen, "The complexity of the generalized Lloyd-max problem," IEEE Transactions on Information Theory, vol. 28, no. 2, pp. 255-256, 1982.

[19] K. S. Al-Sultan, "A Tabu search approach to the clustering problem," Pattern Recognition, vol. 28, no. 9, pp. 1443-1451, 1995.

[20] S. Z. Selim and K. Al-Sultan, "A simulated annealing algorithm for the clustering problem," Pattern Recognition, vol. 24, no. 10, pp. 1003-1008, 1991.

[21] C. A. Murthy and N. Chowdhury, "In search of optimal clusters using genetic algorithms," Pattern Recognition Letters, vol. 17, no. 8, pp. 825-832, 1996.

[22] J. Kennedy and R. Eberhart, "Particle swarm optimization," in Proceedings of the IEEE International Conference on Neural Networks, vol. 4, pp. 1942-1948, IEEE, Perth, Australia, December 1995.

[23] J. L. Fernández Martínez, E. Garcia-Gonzalo, and J. P. Fernández-Alvarez, "Theoretical analysis of particle swarm trajectories through a mechanical analogy," International Journal of Computational Intelligence Research, vol. 4, no. 2, pp. 93-104, 2008.

[24] J. L. Fernández-Martínez and E. García-Gonzalo, "Stochastic stability and numerical analysis of two novel algorithms of the PSO family: PP-GPSO and RR-GPSO," International Journal on Artificial Intelligence Tools, vol. 21, no. 3, Article ID 1240011, 20 pages, 2012.

[25] J. L. Fernández Martínez and E. Garcia-Gonzalo, "Stochastic stability analysis of the linear continuous and discrete PSO models," IEEE Transactions on Evolutionary Computation, vol. 15, no. 3, pp. 405-423, 2011.

[26] J. L. Fernández Martínez, E. García Gonzalo, Z. Fernández Muñiz, and T. Mukerji, "How to design a powerful family of particle swarm optimizers for inverse modelling," Transactions of the Institute of Measurement and Control, vol. 34, no. 6, pp. 705-719, 2012.

[27] A. Hatamlou, S. Abdullah, and M. Hatamlou, "Data clustering using big bang-big crunch algorithm," in Innovative Computing Technology, vol. 241 of Communications in Computer and Information Science, pp. 383-388, 2011.

[28] M. Dorigo, G. Di Caro, and L. M. Gambardella, "Ant algorithms for discrete optimization," Artificial Life, vol. 5, no. 2, pp. 137$172,1999$.

[29] E. Rashedi, H. Nezamabadi-pour, and S. Saryazdi, "GSA: a gravitational search algorithm," Information Sciences, vol. 179, no. 13, pp. 2232-2248, 2009.

[30] A. Hatamlou, S. Abdullah, and Z. Othman, "Gravitational search algorithm with heuristic search for clustering problems," in Proceedings of the 3rd IEEE Conference on Data Mining and Optimization (DMO '11), pp. 190-193, Putrajaya, Malaysia, June 2011.

[31] S. Das, A. Abraham, and A. Konar, "Metaheuristic pattern clustering - an overview," in Metaheuristic Clustering, vol. 178 of Studies in Computational Intelligence, pp. 1-62, Springer, Berlin, Germany, 2009.
[32] S.-H. Liao, P.-H. Chu, and P.-Y. Hsiao, "Data mining techniques and applications-a decade review from 2000 to 2011," Expert Systems with Applications, vol. 39, no. 12, pp. 11303-11311, 2012.

[33] S. J. Nanda and G. Panda, "A survey on nature inspired metaheuristic algorithms for partitional clustering," Swarm and Evolutionary Computation, vol. 16, pp. 1-18, 2014.

[34] D. Simon, "Biogeography-based optimization," IEEE Transactions on Evolutionary Computation, vol. 12, no. 6, pp. 702-713, 2008.

[35] W. Guo, M. Chen, L. Wang, S. S. Ge, and Q. Wu, "Design of migration operators for biogeography-based optimization and markov analysis," Submitted to Information Sciences.

[36] D. Simon, M. Ergezer, and D. Du, "Population distributions in biogeography-based optimization algorithms with elitism," in Proceedings of the IEEE International Conference on Systems, Man and Cybernetics (SMC '09), pp. 991-996, October 2009.

[37] C. L. Blake and C. J. Merz, "UCI repository of machine learning databases," 1998, http://archive.ics.uci.edu/ml/datasets.html.

[38] A. K. Jain, "Data clustering: 50 years beyond K-means," Pattern Recognition Letters, vol. 31, no. 8, pp. 651-666, 2010.

[39] A. Hatamlou, "Black hole: a new heuristic optimization approach for data clustering," Information Sciences, vol. 222, pp. 175-184, 2013.

[40] S. Rana, S. Jasola, and R. Kumar, "A review on particle swarm optimization algorithms and their applications to data clustering," Artificial Intelligence Review, vol. 35, no. 3, pp. 211-222, 2011.

[41] A. M. Bagirov and J. Yearwood, "A new nonsmooth optimization algorithm for minimum sum-of-squares clustering problems," European Journal of Operational Research, vol. 170, no. 2, pp. 578-596, 2006.

[42] S. Yang, R. Wu, M. Wang, and L. Jiao, "Evolutionary clustering based vector quantization and SPIHT coding for image compression," Pattern Recognition Letters, vol. 31, no. 13, pp. 17731780,2010

[43] M. Ergezer, D. Simon, and D. Du, "Oppositional biogeographybased optimization," in Proceedings of the IEEE International Conference on Systems, Man and Cybernetics (SMC '09), pp. 1009-1014, October 2009.

[44] S. Yazdani, J. Shanbehzadeh, and E. Aminian, "Feature subset selection using constrained binary/integer biogeography-based optimization," ISA Transactions, vol. 52, no. 3, pp. 383-390, 2013.

[45] S. Paterlini and T. Krink, "High performance clustering with differential evolution," in Proceedings of the Congress on Evolutionary Computation (CEC '04), vol. 2, pp. 2004-2011, Piscataway, NJ, USA, June 2004.

[46] P. S. Shelokar, V. K. Jayaraman, and B. D. Kulkarni, "An ant colony approach for clustering," Analytica Chimica Acta, vol. 509, no. 2, pp. 187-195, 2004 


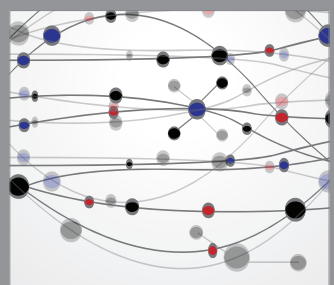

The Scientific World Journal
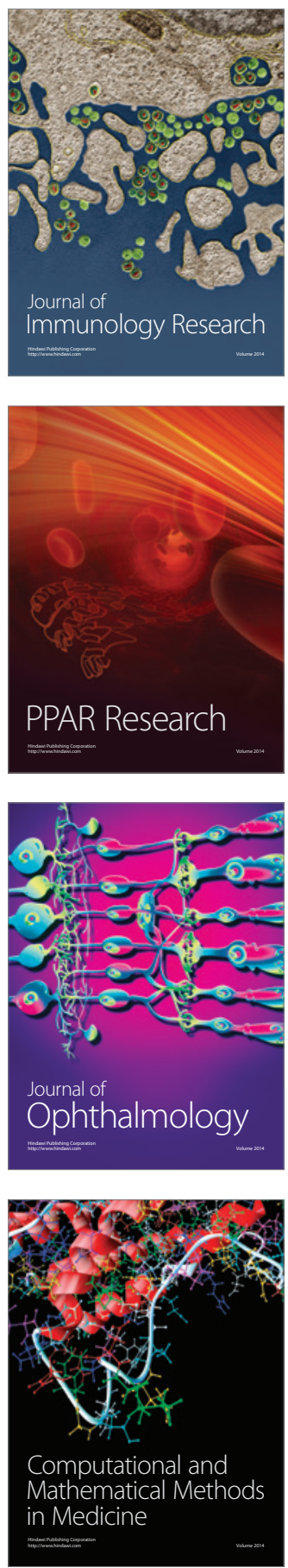

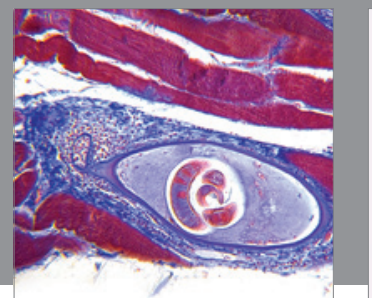

Gastroenterology

Research and Practice
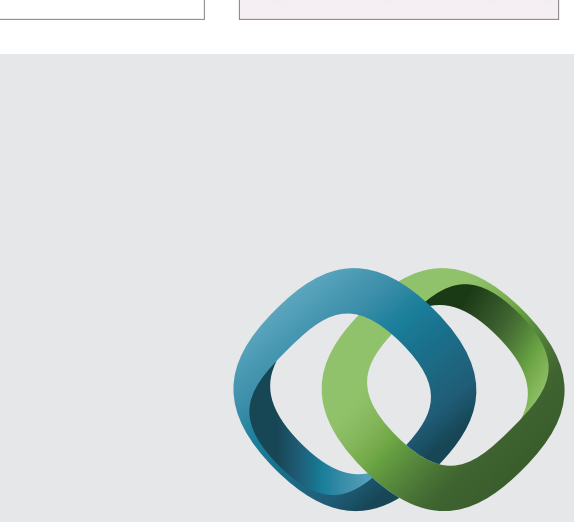

\section{Hindawi}

Submit your manuscripts at

http://www.hindawi.com
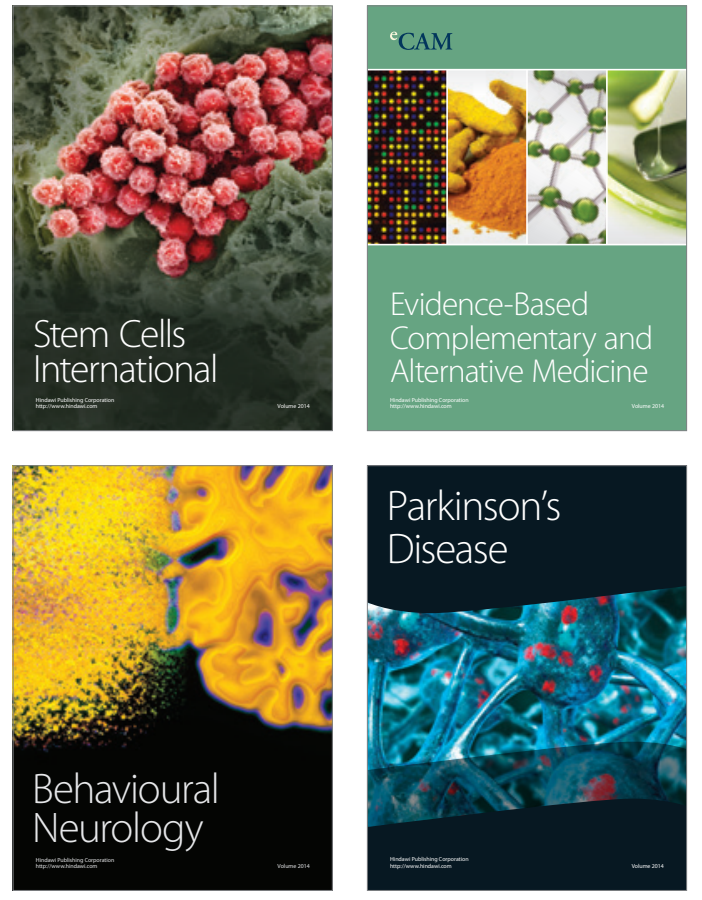
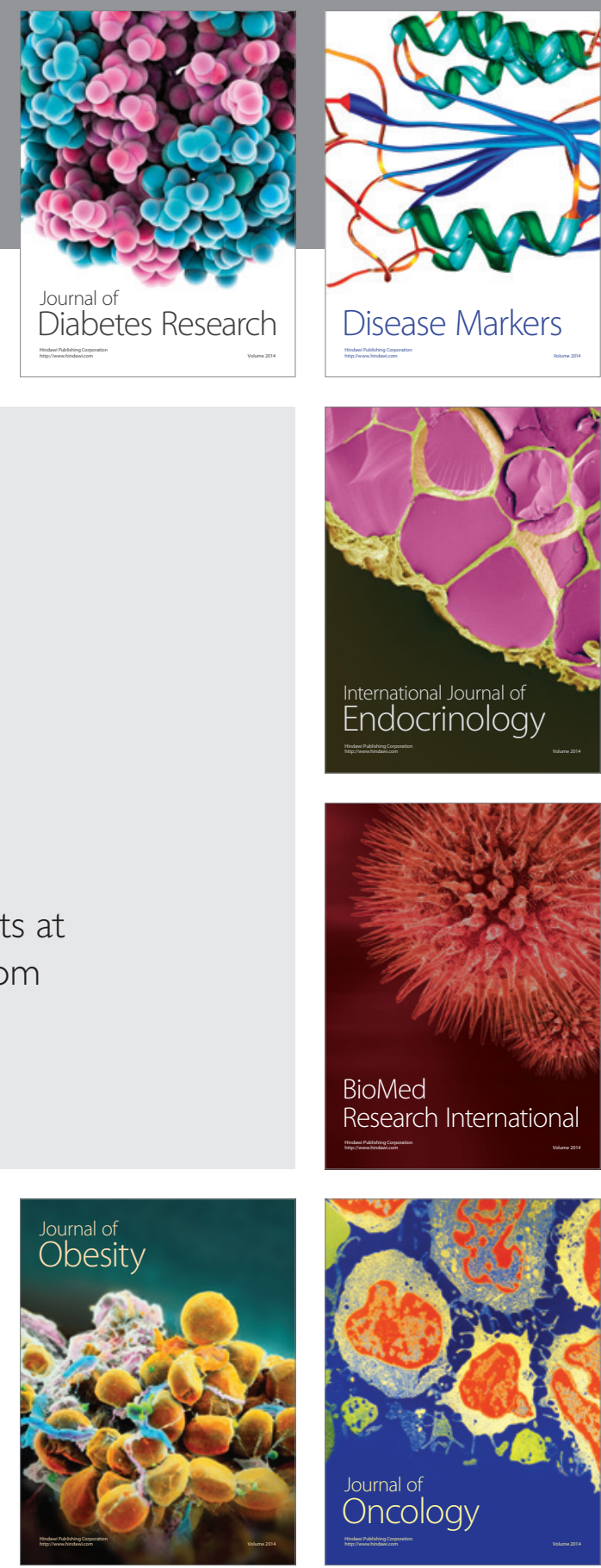

Disease Markers
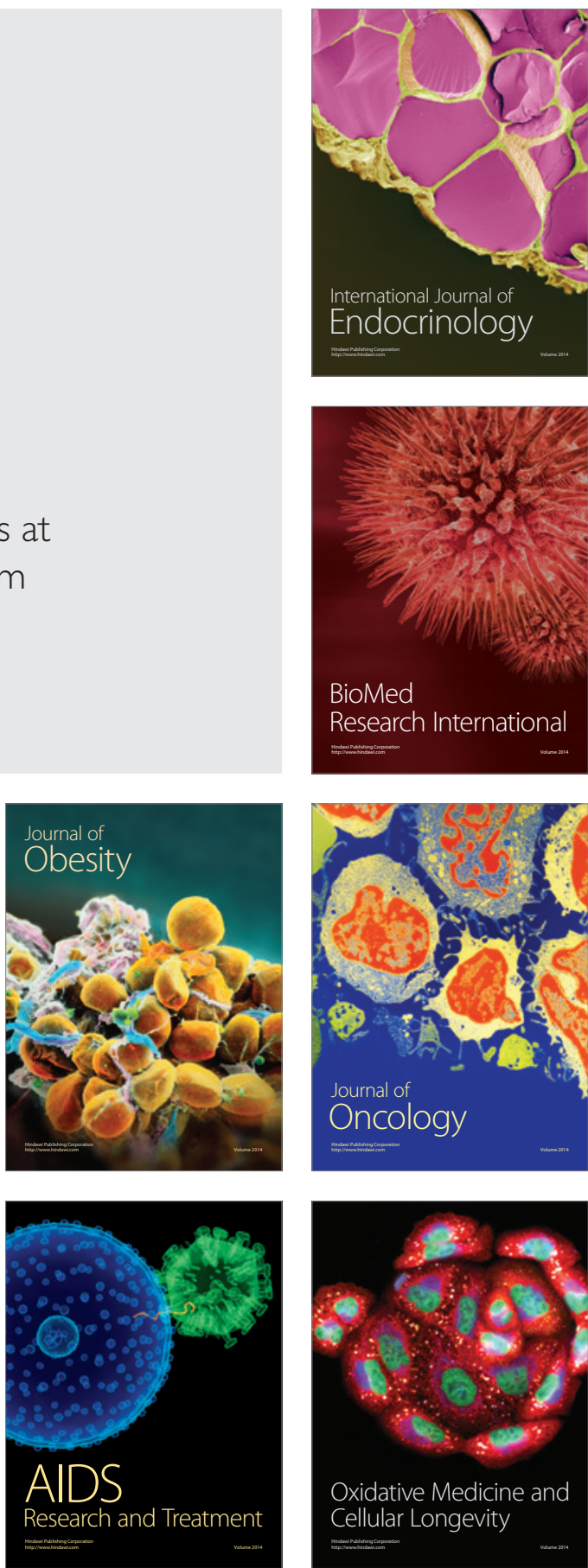